\section{§7. Hybrid Alfvén Resonant Mode Generation in the Magnetosphere-ionosphere Coupling System}

Hiraki, Y. (National Inst. of Polar Res.), Watanabe, T.-H.

Feedback unstable Alfvén waves involving global field-line oscillations and the ionospheric Alfvén resonator (IAR) were comprehensively studied to clarify their properties of frequency dispersion, growth rate, and eigenfunctions. As an extended study of our previous works,${ }^{1)}$ linear eigenmodes of ionospheric feedback instability in the dipole magnetic field $\left(B_{0}\right)$ geometry were analyzed by considering the ionospheric and magnetospheric resonant cavities of the Alfvén velocity $\left(v_{\mathrm{A}}\right)$. The two-field reduced magnetohydrodynamic model,

$$
\begin{aligned}
& \partial_{t} \omega+\boldsymbol{v}_{0} \cdot \nabla_{\perp} \omega=v_{\mathrm{A}}^{2} \nabla_{\|} j_{\|} \\
& \partial_{t} \psi+\boldsymbol{v}_{0} \cdot \nabla_{\perp} \psi+\frac{1}{B_{0}} \nabla_{\|} B_{0} \phi=0,
\end{aligned}
$$

is used to describe shear Alfvén wave dynamics, associated with auroral arcs, in a strongly non-uniform magnetic flux tube; see our paper ${ }^{1)}$ for definition of these variables.

These equations are coupled with the two-fluid equations in the ionosphere as,

$$
\begin{aligned}
& \partial_{t} n_{\mathrm{e}}+\boldsymbol{v}_{0} \cdot \nabla_{\perp} n_{\mathrm{e}}=j_{\|}-R n_{\mathrm{e}} \\
& -\alpha \nabla_{\perp}^{2} \phi+\left(\mu_{\mathrm{P}} \boldsymbol{E}_{0}-\boldsymbol{v}_{0}\right) \cdot \nabla_{\perp} n_{\mathrm{e}}=D \nabla_{\perp}^{2} n_{\mathrm{e}}-j_{\|}(4)
\end{aligned}
$$

yielding the linear dispersion relation for feedback instability; see our paper ${ }^{1)}$ for definition of these variables. Equations (1)-(4) are solved to obtain the eigenfrequency and eigenfunctions of Alfvén waves shown in Figs. 1 and 2.

This study ${ }^{2)}$ discovered that a new mode called here the hybrid Alfvén resonant (HAR) mode can be destabilized in the magnetosphere-ionosphere coupling system with a realistic $v_{\mathrm{A}}$. The HAR mode found in a high frequency range over $0.3 \mathrm{~Hz}$ is caused by coupling of IAR modes $(0.5,1 \mathrm{~Hz}, \ldots)$ with strong dispersion and field line resonances (FLR). The harmonic relation of HAR eigenfrequencies is characterized by a constant frequency shift from those of IAR modes. The three modes (FLR, IAR, and HAR) are robustly found even if effects of two-fluid process and ionospheric collision are taken into account, and thus are anticipated to be detected by magnetic field observations in auroral and polar-cap regions.

1) Hiraki Y., and T.-H. Watanabe: J. Geophys. Res. 116 (2011) A11220.

2) Hiraki Y., and T.-H. Watanabe: Phys. Plasmas 19 (2012) 102904.
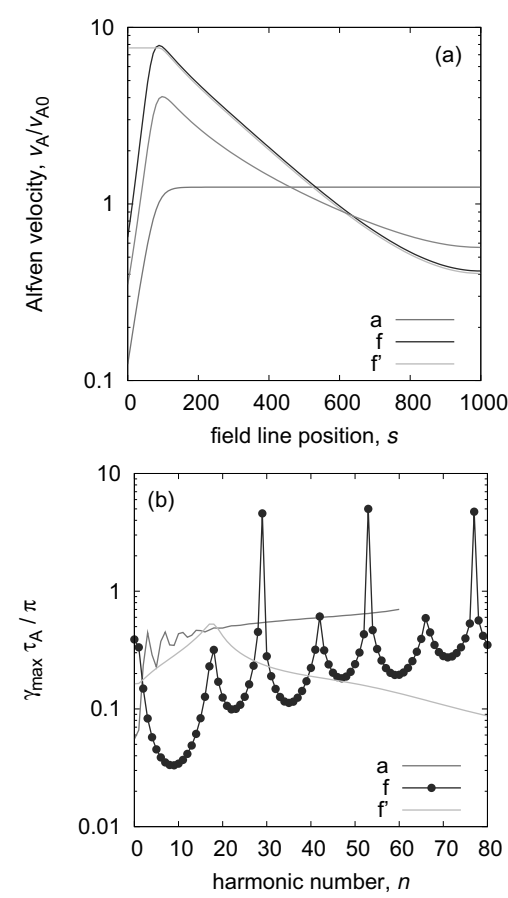

Fig. 1: (a) Alfvén velocity profiles $v_{\mathrm{A}}(s)$ used in this analysis. (b) The maximum growth rate $\gamma_{\max }(n) \tau_{\mathrm{A}} / \pi$ as a function of harmonic number $n ; \gamma \equiv \operatorname{Im}(\Omega)$ with the Alfvén transit time $\tau_{\mathrm{A}}$.
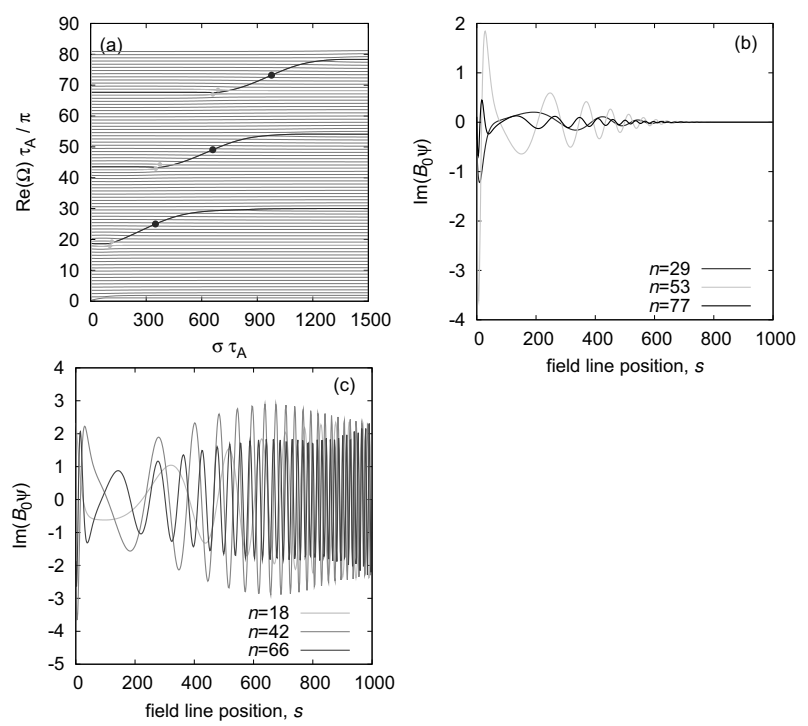

Fig. 2: (a) Real part of eigenfrequency $\operatorname{Re}(\Omega) \tau_{\mathrm{A}} / \pi$ as a function of electric drift frequency $\sigma$ for $v_{\text {A }}$ profile $\mathrm{f}$ in Fig. 1. The harmonics $n=0-80$ are shown. Shown are eigenfunctions $\operatorname{Im}\left(B_{0} \psi\right)$ of (b) IAR $(n=29,53$, and $77)$ and (c) HAR ( $n=18,42$, and 66$)$ modes providing $\gamma_{\max }(n)$. 